\title{
OSGIS \& FOSS in Lbs Design
}

\author{
Shang Zhang ${ }^{1,2,3}$ \\ ${ }^{1}$ Faculty of Information Engineering, China University of \\ Geosciences \\ Beijing, China \\ ${ }^{2}$ College of Computer and Information Technology \\ China Three Gorges University \\ Yichang, China \\ ${ }^{3}$ Zondy Cyber Group Co.,Ltd \\ e-mail: wetoo@163.com
}

\author{
Tingyan Xing, Liufeng Tao \\ Faculty of Information Engineering \\ China University of Geosciences \\ Beijing, China
}

\author{
Fan Zhang \\ Faculty of Information Engineering \\ China University of Geosciences \\ Wuhan,China \\ e-mail: zhangfan0725@aol.com
}

\begin{abstract}
Geographical Information System (GIS) always play an integral role in LBS systems. But it comes with high entry cost. The aim of this paper is to reveal the potential of Free and Open Source Software for GIS in LBS design and practice, with the acceptance and usage of open standards for LBS applications, which provides a possible solution to overcome the high GIS's cost issue. In this paper, the short review of FOSS and OSGIS are given first and then the possibility of LBS design with FOSS are analyzed. By contrasting FOSS with the proprietary software development cycle, and designing a LBS platform based on FOSS middle ware in a new way, this paper proves the possibility of OSGIS could reduce the cost and complexity of LBS design.
\end{abstract}

Keywords- FOSS; LBS; OGIS; Middle ware

\section{INTRODUCTION}

With the development of 3G technology and the Global Navigation Satellite Systems (GNSS), the location information (latitude and longitude coordinates) of mobile end user can be obtained through the mobile communication network which caused a growing interest in location aware services. A location sensing system responsible for locating a mobile user, is a crucial factor for the success of such services. With the prevalence of wireless hotspots and wireless area networks, the use of a wireless network infrastructure as basis for Location Based Service (LBS), which is a platform that provides information services based on the current or a known location, supported by the electronic map platform, and an indoor positioning system becomes a viable option [1].

The increasing demand for commercial LBS has driven scientists to focus on more accurate positioning solutions. It employs accurate, real-time positioning to connect users to points of interest and advises them of the current conditions such as traffic and weather conditions, or provides routing and tracking information using wireless devices. It is important to integrate the Wireless Communication(WC) technologies and the mobile GIS computing technology in order to meet the needs of LBS [2], which is considered one of the most promising applications of GIS.

At same time, Open source strategy is an adequate mean to push current developments within the GIS and Spatial process domain, especially for the ongoing research and development topic "LBS". The availability of Free and Open Source Software (FOSS) for Geographic Information systems (GIS) have increased tremendously in recent years [3].

\section{BIEF REVIEW OF OSGIS AND LBS}

\section{A. FOSS}

Free and open source software (FOSS) or free/libre/open source software (FLOSS) is technically defined as software whose licenses grant users the right to use, copy, study, change, and improve its design for any purpose, and to freely redistribute either the original or modified program without further limitations or royalty payments. This sometimes, preventing users to impose restrictions on other users. In order to make this possible, access to the source code is a necessary condition $[3,4]$.

\section{B. OSGIS}

As the automation of map-based information, GIS technology is rapidly improved and stabilized. Indeed, GIS are becoming an important framework for understanding a dynamic world. The scope of GIS implementation is also extensive - from a desktop implementation to an enterprise operation investing significantly in data storage and processing assets. More and more GIS have been applied in 
national economy and social life, they stand in need of the interoperability and reusability. It's not surprising, then, that a large commercial GIS industry has emerged to provide customers with software tools, training, and source data. However, the cost of large deployments using commercial software can be prohibitive [7].

At the same time their limitations in with the interoperability, portability and data sharing became more obvious. OpenGIS (Open GIS Interoperability Specification) is coming to address the above issues, the specification defines a detailed criteria for GIS software developers. In these cases, open source applications have an equal potential for large-scale implementation across a broad range of platforms, but they can be far more cost-effective and flexible [4].

The OGC(Open GIS Consortium) is an attempt to create open standards for geo-spatial data and systems that further the cause of interoperability. For the sake of the reusability, a component-ware or distributed objects is a new approach to software development. Component is a software pieces that can be assembled into various applications. Microsoft's OLE/COM and the Object Management Group's CORBA model are currently the two dominant model for software component. To satisfy the such trends of the information system, the open community have been developing the component-based Open GIS software system [7].

As long as strictly abide by the OpenGIS standards, software engineers will develop the product which can meet the requirement and inter-operate well, that is, the product can achieve the data and function sharing between GIS and LBS.

\section{Brief State Fo The Art Of LBS}

Location Based Services (LBS) have become a popular technology to retrieve information about the surroundings of a mobile user. A Location Based Service needs the current location of the user in order to provide information about his surroundings, to compute the route from his location to his destination, and provides other information related to location [2].

Location information (latitude-longitude etc.) is dynamically determined by various positioning technologies such as Global Positioning System(GPS), CELL-ID and Enhanced Observed Time Difference(E-OTD). The location information is transmitted back to mobile service provider's server for location-based analysis and integrates location intelligence into their mobile services [9].

Performing spatial query and analysis has always been an integral part of LBS and it is one of the most challenging tasks in providing LBS.

Applications of LBS can be categorized into two classes: personal applications and commercial ones. LBS for commercial use can be seen in material flow industry, public safety and police. Since there are different requirements in different industries as well as in different units of the same industry, the service providers should have different solutions, business flows and price plans that meet different customer's needs. LBS for personal use include emergency aid, navigation and positioning, public transportation routing and transferring, tracking and monitoring, site nearby information gathering, entertaining and gaming, etc $[9,10]$.

\section{PossibILITY Fo DEVELOPING LBS BASEd ON OSGIS}

\section{A. Context}

The goal of OpenGIS specification is to solve the geodata standard with considering the features of different embedded sharing and interoperable geo-processing.

GIS(Geography Information System)plays an important role in LBS, providing map service, routing service, geocoding/decoding, computing and analysis. Map service is the most important service provided by GIS, with which map can be displayed well and LBS information can be shown conveniently. Map is the most useful tool to assist users to define positions and show the result of LBS [9].

However, several drawbacks hindered communities choose commercial software for implementing GIS projects. Firstly, organizations lacking of enough financial resource can not afford the cost of purchasing software. Further more, commercial packages typically are large applications and tightly integration, organizations should pay for the whole package though limited functional components are necessary. Third, by virtue of these packages are with complex logics and have a steep learning curve, professionals in software operation and maintenance are required. So many organizations have to give up their intentions of deploying geographic data and geo-processing capabilities over Internet [7].

Providing GIS-supported LBS poses high entry barriers such as GIS infrastructure and expertise, database maintenance and expensive mapping datasets. This requires huge initial investment from the service provider and application developer [1].

However, the fast pace of technology changes and nonexistent of standards in the telecommunications industry inevitably leads to plenty of false starts and wasted effort in LBS development [13]. The difficulty, high cost and technical complexity of developing LBS has always been underestimated, which leads to the downright disappointment of pre-bubble promises on LBS deployment.

\section{B. System Analysis}

The approach here is to examine the use of existing features of GIS to support development of more advanced LBSs. There are several important aspects of a GIS, which have to be analyzed when trying to create innovations about the extra value that GIS can offer to LBS. They are: geographic data collection and conversions,geographic data management, geographic data analysis, and geographic data presentation. Considering data collection and conversion, the knowledge and methods of GIS can support LBS development [9].

Among GIS a rich amount of different data collection and digitization methods are used, and new methods are all the time developed. GPS was a remarkable advancement in its time,and at the moment laser scanning gives a lot of new possibilities especially for 3D model generation. GIS data 
are not only made of points, but also lines and curves, polygons and volumes as well as data modeled as grid structure. Coordinates make the instrument to integrate any data set and convert them into one model of the reality.GIS is not only 2D; 3D models and virtual realities can offer an interesting alternative for a traditional guide map. Also LBS applications can put this to use [5,8].

Considering geographic data management, the geographic databases are nowadays open and have standard interfaces. In theory, there is no problem in distributed geoprocessing and interoperability. Of course it takes its time to stabilize the situation so that geographic data can be transferred as easily and processed as typically as any regular non-spatial data type. Spatial relational data management systems and graphical/spatial extensions of standard development environments, like PostGIS, a spatial extensions for the PostgreSQL database, enabling geospatial queries, make this possible. Also some other features of geographic databases are interesting from the point of view of LBS applications. For example so-called multiple representations in databases closely connected with the generalization issue [10].

Considering geographic analysis, the LBS can get support from the strong sub-field of GIS in spatial processing of data by using so-called GIS analysis tools such as FDO, which a API between GIS application and sources; for manipulating, defining and analyzing geospatial data, based on spatial algorithms and data structures. There is much in common between spatial algorithms and computational geometry, but there is also a lot of knowledge developed specifically for GIS. GIS analysis is at the moment making a major transition from simple geometry and topology based analysis tools towards more advanced computational methods called Geo-Computation, as well as spatial modeling. The goals of Geo-Computation include development of more advanced analysis tools for GIS, based on classical and modern theories. The theories involve: mathematical modeling, optimization, simulation, statistics, fuzzy modeling, cellular automata, knowledge based systems, fractal analysis, neural computing, spatial multimedia,visualization, and genetic programming. Considering geographic data presentation, the LBSs can gain from the advances in GIS. The traditional visualization method, 2D map, has grown into interactive, animative, 3D and 4D virtual models. In mobile applications map generalization and presentation knowledge has experienced a renaissance $[9,10,12]$.

LBS system development is also relying on GIS standardization. Standards of OpenGIS Consortium (OpenGIS) for spatial data modeling and processing are extensively used for increasing interoperability. Standardization has occurred also via ISO (ISO 21 1TC). OpenGIS and ISO are working in parallel. Development of an XML-based GML(Geography Markup Language) is also a significant attempt towards interoperability of different systems and indicates the high importance of LBS [7].

\section{A Protype Lbs SerVice Platform Design}

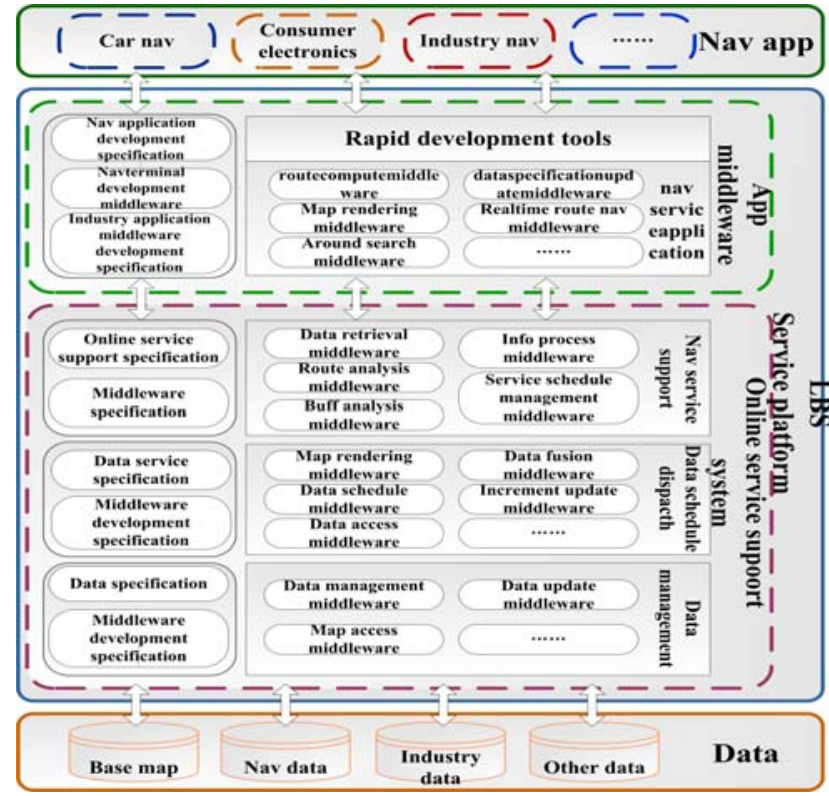

Figure 1. the architecture of LBS service platform

Data is the foundation of all applications and services. The uniqueness and accuracy are the important characteristics of a geographic entity, therefore, in this LBS platform differences and ambiguity caused by the multiple data sources must be overcomed. This platform in the LBS based service is to deal with all kinds of basic data, including the navigation electronic maps of all kinds of manufacturers, all kinds of industry data and remote sensing image data. LBS based service mainly has two parts, one is an online service support system, another is a middle-ware system. The design, implementation, operation of these two parts require rigorous data standard and constraint service specification.

\section{A. Online Support Service System}

For online service support system,based on the data management module, which conduct the most basic data management, multi-source data fusion, data processing and distribution, the diversity of data services, third-party data service support and so on. The design is to build a quick compatibility and integration of a variety of data sources of standard geographic data management system.

In establishing such a unified geographic data management system, flexible scheduling and data dispatch module are needed. Due to the diversity of the LBS service and the growing demand for the industry, different service requirements for data format is also diverse. Through data scheduling and dispatch module, and according to the data content choice, the user can specify its own data format needs,and carry on the content data release, data integration and updating. Therefore, all data are standard unified and constant. The diversification is also the important guarantee of accurate LBS service. According to industry application 
and characteristics of the public service, while through the data access middle-ware and services middle-ware interface, the LBS platform can be customized.

The Nav service support module is a platform to support various types of online services, it mainly includes various navigation electronic map service functions, such as spatial analysis,buffer analysis, data scheduling, information processing, online service application, user management, and other functions. Those functions are designed to provide online function module for the user of third party, and also could for the middle-ware design and development, which can be composed for all kinds of navigation application.

\section{B. The Middle-Ware Platform}

Middle-ware system is the core of LBS service, which throughout the underlying data management to online services support, and the application development, middleware technology process.

The service platform within the various types of middleware. In order to achieve structure flexible and functional independence, function modules are based on middle-ware implementation, which are basement of the infrastructure of the services platform.

In the field of electronic map navigation application, middle-ware is the main form and content of navigation terminal development, their complementation are completely followed by the specification shown in the Firgure-1. In development, the use of middle-ware can have better classification of all kinds of functions and extensions, which can assist the user to build and deploy the LBS services.

Otherwise the rapid development tools are, moreover, providing an important feature of LBS platform, which increase the difficulty of software development, shorten the development cycle, solve the problem of multiplication differences. The tool can support multiple mobile devices in terms of building type development, the development of the core functionality can be implemented directly by the middle-ware in the form of drag and drop. Rapid development tools make full use of the navigation terminal development, middle-ware and industry application which could improve the efficiency of software development, enhances its extensibility.

\section{CONCLUSION}

LBS public service will also change the ordinary way of life, but also bring enormous business opportunities. At present, the high cost of service influence the LBS public service. The development and application of FOSS or OSCIG based product will provide LBS service with a high efficiency and low cost.

Based on FOSS/OSGIS, which can absorb scientific and technological achievements in the field of information technology synchronously, keep up with advanced concept of software architecture, so as to promote the development of LBS technology and software.

\section{ACKNOWLEDGMENT}

At the point of finishing this paper, I'd like to express my sincere thanks to all those who have lent me hands in the course of my writing this paper. First of all, I'd like to take this opportunity to show my sincere gratitude to my supervisor, Pro $\mathrm{Wu}$, who has given me so much useful advices on my writing. Secondly, I'd like to express my gratitude to my classmates who offered me references and information on time. Last but not the least, I'd like to thank those leaders, teachers and working staff especially those in the China University of Geosciences.

National science and technology support project (2011BAH06B04)-"Reaserch of geospatial information toolsetweb services platform"

\section{REFERENCES}

[1] Sabbour, A.A., "WiGuide: Indoor System for LBS”. 2007, German University in Cairo.

[2] Sadoun, B.; Al-Bayari, O., "LBS and GIS Technology Combination and Applications," Computer Systems and Applications, 2007. AICCSA '07. IEEE/ACS International Conference on , vol., no., pp.578,583, 13-16 May 2007

[3] https://en.wikipedia.org/wiki/Free_and_open-source_software

[4] Kon, F., et al. "Free and Open Source Software Development and Research: Opportunities for Software Engineering”. in Software Engineering (SBES), 2011 25th Brazilian Symposium on. 2011.

[5] Park, M.H., H.C. Kim and S.J. Lee. "Implementation results and service examples of GPS-Tag for indoor LBS and message service in Advanced Communication Technology” (ICACT), 2013 15th International Conference on. 2013.

[6] JingDeng,XinqiZheng,LinaLv,“A Method for Service Oriented WebGIS Construction by Integrating OpenSource GIS”,978-1-61284848-8/11@2011IEEE

[7] David A. Garbin and James L. Fisher, Noblis, “Open Source for Enterprise Geographic Information Systems”, IT Pro November/December $2010 \quad$ pp. 38-45.

[8] Isabelle Kiener,"Development of a Location Based Service for a University Campus",School of Surveying and Spatial Information Systems, The University of New South Wales, Sydney, Australia, February 2003

[9] Xiaolin, W., P. Xiao and L. Yingwei. "LBS-p: A LBS Platform Supporting Online Map Services”. in Vehicular Technology Conference Fall (VTC 2010-Fall), 2010 IEEE 72nd. 2010.

[10] Zhang, L., L. Kunhui and W. Ye. "Location-Based Mobile Search: And the Application of Searching for Hotels". in Instrumentation, Measurement, Computer, Communication and Control (IMCCC), 2012 Second International Conference on. 2012.

[11] Jaegeol Yim,Ilseok Ko,Ilseok Ko,"Implementation of a Prototype Positioning System for LBS on U-campus "Journal of Universal Computer Science, vol. 14, no. 14 (2008), 2381-2399

[12] Jingbin Liu *, Ruizhi Chen, Ling Pei,Robert Guinness and Heidi Kuusniemi “A Hybrid Smartphone Indoor Positioning Solution for Mobile LBS ”Sensors 2012, 12, pp:17208-17233

[13] C.M. Wang, Z.M. Wang and L.Z. Zhu,"Problems and Methods in the Location-Based Services,”Geomatics \& spacial information technology, vol. 32, June 2007, pp. 124-127 